\title{
Continuum Elastic Theory of Adsorbate Vibrational Relaxation
}

\author{
Steven P. Lewis, ${ }^{a}$ M. V. Pykhtin, ${ }^{b}$ E. J. Mele, ${ }^{b}$ and Andrew M. Rappe ${ }^{a}$ \\ Department of Chemistry, ${ }^{a}$ Department of Physics, ${ }^{b}$ and \\ Laboratory for Research on the Structure of Matter, ${ }^{a, b}$ \\ University of Pennsylvania, Philadelphia, PA 19104.
}

(May 20, 2018)

\begin{abstract}
An analytical theory is presented for the damping of low-frequency adsorbate vibrations via resonant coupling to the substrate phonons. The system is treated classically, with the substrate modeled as a semi-infinite elastic continuum and the adsorbate overlayer modeled as an array of point masses connected to the surface by harmonic springs. The theory provides a simple expression for the relaxation rate in terms of fundamental parameters of the system: $\gamma=m \bar{\omega}_{0}^{2} / A_{c} \rho c_{T}$, where $m$ is the adsorbate mass, $\bar{\omega}_{0}$ is the measured frequency, $A_{c}$ is the overlayer unit-cell area, and $\rho$ and $c_{T}$ are the substrate mass density and transverse speed of sound, respectively. This expression is strongly coverage dependent, and predicts relaxation rates in excellent quantitative agreement with available experiments. For a half-monolayer of carbon monoxide on the copper (100) surface, the predicted damping rate of in-plane frustrated translations is $0.50 \times 10^{12} \mathrm{~s}^{-1}$, as compared to the experimental value of $(0.43 \pm 0.07) \times 10^{12} \mathrm{~s}^{-1}$. Furthermore it is shown that, for all coverages presently accessible to experiment, adsorbate motions exhibit collective effects which cannot be treated as stemming from isolated oscillators.
\end{abstract}

Low-frequency adsorbate vibrations have important consequences in many fundamental chemical processes at surfaces, including reactivity, surface diffusion, and desorption. Understanding the dynamical nature of the low-frequency modes can provide important insights into the physical behavior of these processes. When the frequency of an adsorbate mode is so low that it lies within the phonon band of the substrate, it can resonantly mix with the phonons to form new normal modes with composite adsorbate- and bulk-like character. Thus the adsorbate mode can be viewed as developing a finite lifetime by decaying into propagating bulk elastic waves. This resonant coupling is an important mechanism in understanding energy relaxation processes at surfaces. It has been shown, for example, to dominate the relaxation dypamics of the in-plane frustrated translational motion of a carbon monoxide adlayer on the (100) surface of copper.

In this article, we present an analytical theory of the resonant coupling of an adsorbate overlayer to its substrate. This theory results in simple mathematical expressions for the vibrational damping rate and the renormalized vibrational frequency of the adlayer, in terms of fundamental properties of the system, such as the mass density and speed of sound of the substrate, the mass of the adsorbate molecules, and the adsorbate vibrational frequency. When applied to systems for which there are experimental data, these expressions give quantitatively accurate results, thus verifying their predictive power.

In this theory, we model the substrate as a flat, isotropic, semi-infinite, classical elastic continuum. The elasticcontinuum approximation is valid when considering adsorbate modes lying in the frequency range of long-wavelength substrate acoustic phonons, where the dispersion can be accurately modeled with a Debye spectrum. Furthermore, the choice of a flat and isotropic substrate is particularly appropriate for low-index surfaces of close-packed materials. Thus, this theory is especially applicable to the in-plane frustrated-translational modes of molecules on low-index metal surfaces. These modes typically have frequencies in the acoustic range of the substrate. The molecular overlayer is modeled as a two-dimensional periodic lattice (or net) of identical, classical point masses, each connected to the surface of the substrate by a classical harmonic spring.

The theory presented here builds on the earlier work of Persson and Ryberg (PR), 2 who examined resonant vibrational decay for the case of a single, isolated adsorbate coupled to an elastic substrate. They deduced the following expression for the damping rate:

$$
\gamma=\frac{1}{8 \pi} \frac{m}{\rho}\left(\frac{\omega_{0}}{c_{T}}\right)^{3} \omega_{0} \xi,
$$

where $\omega_{0}$ is the resonance frequency, $m$ is the adsorbate mass, $\rho$ is the substrate mass density, $c_{T}$ is the transverse speed of sound in the substrate, and $\xi$ is a dimensionless constant of order unity which depends only on the ratio of the transverse and longitudinal speeds of sound. We show below, however, that a dense layer of adsorbates behaves fundamentally differently. Collective motion of the adlayer strongly enhances the coupling efficiency compared to the isolated case, whereas interference effects restrict the phase space of substrate modes available for coupling. One 
might imagine that both limits could be achieved experimentally by varying the surface coverage. However, we also show below that the dense-adlayer limit holds for an enormous range of coverages. The isolated-adsorbate limit does not set in until coverages well below that which can be observed by current experimental techniques.

We begin by developing the theory in its most general form, which includes both the dense-adlayer and the isolatedadsorbate extremes as limiting cases. Afterwards, we indicate how to deduce the PR result and then focus in detail on the dense-adlayer limit, as this is the experimentally realizable case. In a forthcoming paper, we will address the general result in greater detail, examining the cross-over behavior between the two extremes.

Consider a semi-infinite, classical elastic medium described by the displacement field $\boldsymbol{u}(\boldsymbol{x}, t)$ and occupying the halfspace $z>0$. Its surface is the $x y$-plane. (In this paper three-dimensional vectors are denoted in boldface, whereas two-dimensional vectors on the surface are designated with arrows.) The surface is covered by a two-dimensional periodic array of point masses $m$ attached at lattice sites $\left\{\vec{R}_{i}\right\}$ by classical harmonic springs of bare frequency $\omega_{0}$ (i.e., spring constant $m \omega_{0}^{2}$ ). Let $s_{i}(t)$ denote the displacement coordinate of the $i$ th adsorbate from its equilibrium position. In general, the polarization of $s_{i}$ can have both in-plane and normal components. However, for simplicity, we consider $s_{i}$ to be fully $x$-polarized. It can be shown, in fact, that the mathematical formulas describing the dynamical response of in-plane and normal motion are identical up to numerical constants of order unity. Furthermore, $x$ - and $y$-polarized motion are degenerate by symmetry.

The equation of motion for the $i$ th adsorbate is given (in the frequency domain) by:

$$
-m \omega^{2} s_{i}(\omega)+m \omega_{0}^{2}\left[s_{i}(\omega)-u_{x}\left(\vec{R}_{i}, \omega\right)\right]=f_{i}(\omega),
$$

where $f_{i}(\omega)$ is the $x$-component of an external driving force on the $i$ th adsorbate. If the substrate were rigid (i.e., $\boldsymbol{u}(\boldsymbol{x}, \omega) \equiv 0)$, the adsorbates would clearly undergo simple harmonic motion at frequency $\omega_{0}$. However an elastic substrate couples to the adsorbate motion, thereby damping the vibration and renormalizing its frequency. The influence of the coupling on the adsorbate dynamics can be described mathematically by the complex poles of the adsorbate response function, $\chi\left(\vec{R}_{i}, \omega\right)$, defined by

$$
s_{i}(\omega)=\chi\left(\vec{R}_{i}, \omega\right) f_{i}(\omega) .
$$

To determine $\chi\left(\vec{R}_{i}, \omega\right)$, we first integrate out the substrate degrees of freedom, $u_{x}(\vec{x}, \omega)$.

The equation of motion for an elastic substrate subject to an external driving force, $\boldsymbol{a}(\vec{x}, \omega)$, at the surface is $\vec{B}$

$$
-\omega^{2} \boldsymbol{u}(\boldsymbol{x}, \omega)-c_{L}^{2} \nabla(\nabla \cdot \boldsymbol{u}(\boldsymbol{x}, \omega))+c_{T}^{2} \nabla \times(\nabla \times \boldsymbol{u}(\boldsymbol{x}, \omega))=\delta(z) \boldsymbol{a}(\vec{x}, \omega) / \rho,
$$

where $c_{L}$ and $c_{T}$ are the longitudinal and transverse speeds of sound, respectively, specifying the elastic properties of the material. The surface driving force is provided by the oscillating adsorbates, and thus is given by

$$
\begin{aligned}
a_{x}(\vec{x}, \omega) & =\sum_{i} m \omega_{0}^{2}\left[s_{i}(\omega)-u_{x}\left(\vec{R}_{i}, \omega\right)\right] \delta\left(\vec{x}-\vec{R}_{i}\right) \\
a_{y, z}(\vec{x}, \omega) & =0 .
\end{aligned}
$$

The solution to Eqs. (4) and (5) is given formally by

$$
u_{\alpha}(\vec{x}, z ; \omega)=\sum_{\beta} \int D_{\alpha \beta}\left(\vec{x}, z ; \vec{x}^{\prime}, z^{\prime} ; \omega\right) a_{\beta}\left(\vec{x}^{\prime}, z^{\prime} ; \omega\right) d^{2} x^{\prime} d z^{\prime}
$$

where $\alpha$ and $\beta$ run over Cartesian directions, and $D_{\alpha \beta}\left(\vec{x}, z ; \vec{x}^{\prime}, z^{\prime} ; \omega\right)$ is the Green's function of the elastic substrate, defined as the $\alpha$-component of the solution to Eq. (4) with source term $\delta^{2}\left(\vec{x}-\vec{x}^{\prime}\right) \delta\left(z-z^{\prime}\right) / \rho$ in the $\beta$-direction and stress-free boundary conditions. Several simplifications can be made for the present problem. First, the driving force, $\boldsymbol{a}$, exists only at the surface and is $x$-polarized. Furthermore, according to Eq. (2), only the $x$-component of $\boldsymbol{u}(\boldsymbol{x}, \omega)$ at the surface is of interest. Finally, translational symmetry parallel to the surface means that $D_{\alpha \beta}$ can only depend on the difference $\vec{x}-\vec{x}^{\prime}$. Thus, Eq. (6) becomes

$$
u_{x}(\vec{x}, \omega)=\int D_{x x}\left(\vec{x}-\vec{x}^{\prime}, \omega\right) a_{x}\left(\vec{x}^{\prime}, \omega\right) d^{2} x^{\prime},
$$

where the $z$ and $z^{\prime}$ arguments are zero and have been suppressed for convenience. In reciprocal space,

$$
u_{x}(\vec{k}, \omega)=D_{x x}(\vec{k}, \omega) a_{x}(\vec{k}, \omega) .
$$


Because of the two-dimensional periodicity of the adsorbate lattice, the stationary solutions to both the substrate and adsorbate equations of motion must obey Bloch's Theorem. Namely,

$$
\begin{aligned}
u_{x}\left(\vec{R}_{i}, \omega\right) & =u_{x}(0, \omega) e^{i \vec{q} \cdot \vec{R}_{i}} \\
s_{i}(\omega) & =s_{0}(\omega) e^{i \vec{q} \cdot \vec{R}_{i}},
\end{aligned}
$$

where $\vec{q}$ is a wavevector in the first Brillouin zone of the adsorbate lattice. Combining Eqs. (5) and (9) and Fourier transforming gives

$$
\begin{aligned}
a_{x}(\vec{k}, \omega) & =m \omega_{0}^{2}\left[s_{0}(\omega)-u_{x}(0, \omega)\right] \sum_{i} e^{i(\vec{q}-\vec{k}) \cdot \vec{R}_{i}} \\
& =m \omega_{0}^{2}\left[s_{0}(\omega)-u_{x}(0, \omega)\right] \frac{(2 \pi)^{2}}{A_{c}} \sum_{\vec{G}} \delta(\vec{q}-\vec{k}+\vec{G}),
\end{aligned}
$$

where $A_{c}$ is the area of the surface unit cell, and $\{\vec{G}\}$ is the set of reciprocal lattice vectors of the overlayer structure.

Equation (10) demonstrates that a coherent excitation of an adsorbate overlayer cannot couple to all resonant substrate modes, in contrast to the case of an isolated adsorbatel 6 Rather, the lattice imposes a selection rule. Interference among the adsorbates in the overlayer prevents energy from being radiated into any substrate mode whose in-plane wavevector component is not commensurate with the adsorbate reciprocal lattice. Furthermore, the factor of $1 / A_{c}$ in Eq. (10) shows that dense adlayers drive the substrate more strongly than diffuse adlayers, or, in the extreme limit, than an isolated adsorbate. Thus the coupling is more efficient for a dense overlayer and leads to a faster damping, as shown below.

Combining Eqs. (8) and (10), inverse Fourier transforming, and evaluating the result at $\vec{x}=\vec{R}_{i}$ gives a simple expression directly relating the substrate and adsorbate degrees of freedom:

$$
u_{x}\left(\vec{R}_{i}, \omega\right)=\left[s_{i}(\omega)-u_{x}\left(\vec{R}_{i}, \omega\right)\right] \frac{m \omega_{0}^{2}}{A_{c}} \sum_{\vec{G}} D_{x x}(\vec{q}+\vec{G}, \omega) .
$$

The response function, $\chi(\vec{q}, \omega)$, defined as the reciprocal-space version of the function appearing in Eq. (3), can be expressed in terms of the substrate Green's function, $D_{x x}(\vec{q}, \omega)$, and fundamental parameters of the system by combining Eqs. (2) and (11), and rearranging the result to be in the form of Eq. (3). Thus,

$$
\begin{aligned}
\chi(\vec{q}, \omega) & =\left[-m \omega^{2}+\frac{m \omega_{0}^{2}}{1+T(\vec{q}, \omega)}\right]^{-1} \\
& =\left[-m \omega^{2}+m \omega_{0}^{2} \frac{1+R(\vec{q}, \omega)}{(1+R(\vec{q}, \omega))^{2}+I^{2}(\vec{q}, \omega)}-i \omega_{0} \frac{m \omega_{0} I(\vec{q}, \omega)}{(1+R(\vec{q}, \omega))^{2}+I^{2}(\vec{q}, \omega)}\right]^{-1},
\end{aligned}
$$

where

$$
T(\vec{q}, \omega)=\frac{m \omega_{0}^{2}}{A_{c}} \sum_{\vec{G}} D_{x x}(\vec{q}+\vec{G}, \omega),
$$

and $R(\vec{q}, \omega)$ and $I(\vec{q}, \omega)$ are the real and imaginary parts, respectively, of $T(\vec{q}, \omega)$. The isolated-adsorbate extreme is obtained from Eq. (13) in the limit of infinitely long overlayer lattice vectors. The Briflouin zone area, $(2 \pi)^{2} / A_{c}$, becomes an infinitesimal and the sum over reciprocal lattice vectors becomes an integral:t

$$
T(\omega)=m \omega_{0}^{2} \int \frac{d^{2} q}{(2 \pi)^{2}} D_{x x}(\vec{q}, \omega) .
$$

The imaginary part of $\chi(\vec{q}, \omega), \operatorname{Im}[\chi(\vec{q}, \omega)]$, is the measurable absorption spectrum. If we assume that $T(\vec{q}, \omega)$ is sufficiently smooth and that the damping rate, $\gamma$, is much smaller than the resonant frequency, $\operatorname{then} \operatorname{Im}[\chi(\vec{q}, \omega)]$ is a sharply peaked function centered at the renormalized frequency $\bar{\omega}_{0}$ (the experimentally measured frequency) and having characteristic width $\gamma$. Under these conditions, we can approximate $\omega \approx \omega_{0}$, which gives $\chi(\vec{q}, \omega)$ the form of a damped harmonic oscillator:

$$
\chi(\vec{q}, \omega) \approx\left[-m \omega^{2}+m \bar{\omega}_{0}^{2}-i \omega m \gamma\right]^{-1},
$$


where

$$
\begin{aligned}
\bar{\omega}_{0}^{2} & =\omega_{0}^{2} \frac{1+R\left(\vec{q}, \omega_{0}\right)}{\left(1+R\left(\vec{q}, \omega_{0}\right)\right)^{2}+I^{2}\left(\vec{q}, \omega_{0}\right)} \\
\gamma & =\frac{\omega_{0} I\left(\vec{q}, \omega_{0}\right)}{\left(1+R\left(\vec{q}, \omega_{0}\right)\right)^{2}+I^{2}\left(\vec{q}, \omega_{0}\right)} .
\end{aligned}
$$

For the remainder of this paper we assume that dispersion in the plane is negligible. This assumption is governed by the length scale for inter-adsorbate interactions, and is expected to be valid for all but the highest surface coverages. Thus all analyses are made for the $q=0$ limit. This restriction could be lifted without difficulty, if warranted.

Determining the dynamical response of the adlayer has been reduced to solving for the Green's function of a flat, semi-infinite, isotropic elastic medium. This has been carried out in great detail by Maradudin, et al.5 Evaluating their expressions for $D_{x x}$ at $z=z^{\prime}=0$ gives

$$
D_{x x}(\vec{k}, \omega)=-i\left[\frac{k_{y}^{2}}{k^{2} \beta p_{T}}+\frac{k_{x}^{2} \omega^{2}}{2 k^{2} c_{T}^{2}} \frac{p_{T}}{p_{L}} \frac{1}{Q\left(k^{2}, \omega\right)}\right],
$$

where

$$
\begin{aligned}
p_{L, T} & =\left[\frac{\omega^{2}}{c_{L, T}^{2}}-k^{2}\right]^{\frac{1}{2}}, \\
Q\left(k^{2}, \omega\right) & =2 \beta k^{2} p_{T}+\frac{\left(p_{T}^{2}-k^{2}\right)}{2 p_{L}}\left[(\alpha-\beta) k^{2}+(\alpha+\beta) p_{L}^{2}\right],
\end{aligned}
$$

and $\alpha=\rho\left(c_{L}^{2}-c_{T}^{2}\right)$ and $\beta=\rho c_{T}^{2}$. Equations (18)-(20) use the same naming conventions as in Appendix B of PR. 1 The analysis that has been presented so far for $x$-polarized adsorbate motion also holds for $z$-polarized motion, provided that $u_{z}$ and $D_{z z}$ replace $u_{x}$ and $D_{x x}$, respectively, throughout, where $D_{z z}$ is given by

$$
D_{z z}(\vec{k}, \omega)=-i \frac{k^{2}+p_{T}^{2}}{2 Q\left(k^{2}, \omega\right)} .
$$

According to Eq. (13) $), D_{x x}(\vec{k}, \omega)$ is to be evaluated only at reciprocal lattice vectors, $\vec{k}=\vec{G}$. This function is pure imaginary unless $p_{T}$ or $p_{L}$ is imaginary. Consider the shortest non-zero reciprocal lattice vector, $\vec{G}_{0}$. Assuming a square adsorbate lattice, $\left|\vec{G}_{0}\right|=2 \pi / a$, where $a$ is the adsorbate lattice constant. The condition for $p_{T, L}$ to be imaginary is $\omega / c_{T, L}<|\vec{G}|$. At $\vec{G}_{0}$, this is equivalent to

$$
a<\lambda_{T, L},
$$

where $\lambda_{T, L}=2 \pi c_{T, L} / \omega$ is the wavelength of a substrate phonon at or near the resonant frequency. However, since the elastic-continuum description of the substrate is only valid at the long-wavelength end of the phonon spectrum, Eq. (22) will be true for the present theory unless the adsorbate lattice is extremely dilute. Suppose, for example, that $\lambda=10 a_{\mathrm{bulk}}$, where $a_{\mathrm{bulk}}$ is the lattice constant of the bulk substrate material. Since surface coverage is given by $\theta=a_{\text {bulk }}^{2} / a^{2}$, Eq. (22) will be violated only for coverages less than $1 \%$, which is below the resolution limit of current surface-sensitive measurement techniques. Thus, for all systems presently accessible to experiment, all nonzero reciprocal lattice vectors contribute only to the real part of $T(\omega)$, and only $\vec{G}=0$ contributes to the imaginary (i.e., damping) part. We call the regime specified by Eq. (22) the "dense adlayer" limit, and it is given in terms of surface coverage by

$$
\theta>\left(\frac{\omega}{2 \omega_{D}}\right)^{2}
$$

where $\omega_{D}=c_{T, L} \pi / a_{\text {bulk }}$ is the substrate Debye frequency.

In the dense adlayer limit, the imaginary part of $T\left(\omega_{0}\right)$ is

$$
I\left(\omega_{0}\right)=\frac{m \omega_{0}^{2}}{A_{c}} D_{x x}\left(0, \omega_{0}\right)=\frac{m \omega_{0}}{A_{c} \rho c_{T}},
$$


TABLE I. Empirical physical parameters for a half-monolayer of carbon monoxide on the copper (100) surface.

\begin{tabular}{lr}
\hline \hline Quantity & Value \\
\hline $\bar{\omega}_{0}\left(10^{12} \mathrm{~s}^{-1}\right)$ & $6.03^{\mathrm{a}}$ \\
$m\left(10^{-23} \mathrm{~g}\right)$ & 4.65 \\
$a\left(10^{-8} \mathrm{~cm}\right)$ & $3.61^{\mathrm{b}}$ \\
$A_{c}=a^{2}\left(10^{-15} \mathrm{~cm}^{2}\right)$ & 1.30 \\
$\rho\left(\mathrm{g} / \mathrm{cm}^{3}\right)$ & $8.93^{\mathrm{b}}$ \\
$c_{T}\left(10^{5} \mathrm{~cm} / \mathrm{s}\right)$ & $2.91^{\mathrm{c}}$ \\
$c_{L}\left(10^{5} \mathrm{~cm} / \mathrm{s}\right)$ & $4.34^{\mathrm{c}}$ \\
\hline \hline
\end{tabular}

${ }^{\mathrm{a}}$ Reference 7

${ }^{\mathrm{b}}$ Reference 6

${ }^{\mathrm{c}}$ Derived from elastic constants in Reference 6

which, when inserted in Eq. (17), gives a damping rate of

$$
\gamma=\frac{m \omega_{0}^{2}}{A_{c} \rho c_{T}} \frac{1}{\left(1+R\left(\omega_{0}\right)\right)^{2}+I^{2}\left(\omega_{0}\right)}=\frac{m \bar{\omega}_{0}^{2}}{A_{c} \rho c_{T}}\left[\frac{1}{1+R\left(\omega_{0}\right)}\right] \approx \frac{m \bar{\omega}_{0}^{2}}{A_{c} \rho c_{T}},
$$

where the expression for $\bar{\omega}_{0}$ in Eq. (16) has been used. Typically, $\left|R\left(\omega_{0}\right)\right|<<1$, so that the bracketed term in Eq. 25 ) can be neglected. Thus the vibrational damping rate of a dense adlayer can be simply computed from empirically available information about the system. Note that the expression for $\gamma$ in Eq. 25) is strongly dependent on the surface coverage through the parameters $A_{c}, \bar{\omega}_{0}$, and $R\left(\omega_{0}\right)$.

The real part of $T\left(\omega_{0}\right)$ is a sum over all non-zero reciprocal lattice vectors. For real systems, the substrate is not a continuum, but rather a discrete lattice. This places an upper bound on the summation limit for the computation of $R\left(\omega_{0}\right)$ : the sum includes all reciprocal lattice vectors of the overlayer structure that lie within the first Brillouin zone of the bare surface. Surface wavevectors outside this region do not correspond to new substrate phonons.

The Green's function $D_{x x}\left(\vec{G}, \omega_{0}\right)$, for $\vec{G} \neq 0$, can be simplified in the dense-adlayer limit by expanding to lowest order in $\left(\omega_{0} / c_{L, T} G\right)^{2}$, where $G=|\vec{G}|$ :

$$
D_{x x}\left(\vec{G}, \omega_{0}\right) \approx \frac{1}{\rho c_{T}^{2} G}\left[\frac{G_{y}^{2}}{G^{2}}+\frac{G_{x}^{2}}{G^{2}} \frac{1}{2\left(1-\left(c_{T} / c_{L}\right)^{2}\right)}\right] .
$$

Since the term in brackets is of order unity, $R\left(\omega_{0}\right)$ is approximately given by

$$
R\left(\omega_{0}\right) \approx \sum_{\vec{G} \neq 0} \frac{m \omega_{0}^{2}}{A_{c} \rho c_{T}^{2} G}
$$

If the overlayer unit cell is small (i.e., high coverage), then the sum will contain only a few reciprocal lattice vectors before reaching the upper bound set by the substrate lattice. Thus, since $\omega_{0} / c_{T} G \ll 1, R\left(\omega_{0}\right)$ will be small. As the unit cell gets larger, more terms are included in the sum. However, the factor $1 / A_{c}$ decreases, keeping $R\left(\omega_{0}\right)$ small.

To test the validity of this theory, we now apply it to a system for which there are experimental data: carbon monoxide on the (100) surface of copper at half-monolayer coverage. The in-plane frustrated translational mode has a measured frequency of $32 \mathrm{~cm}^{-1}$, well yithin the range of long-wavelength copper phonons. Recently, the lifetime of this mode was measured to be $2.3 \pm 0.4 \mathrm{ps}, 0$ which corresponds to a damping rate $(0.43 \pm 0.07) \times 10^{12} \mathrm{~s}^{-1}$. Other parameters for this system are compiled in Table II. Using these data, Eq. (25), with the bracketed term neglected, predicts the frustrated translational damping rate to be $\gamma=0.50 \times 10^{12} \mathrm{~s}^{-1}$, in excellent agreement with the experimental value.

The bracketed term in Eq. (25) produces a small downward correction. To show this we now compute $R\left(\omega_{0}\right)$ using the expression in Eq. (26). The CO overlayer forms a square lattice with $c(2 \times 2)$ symmetry relative to the underlying copper (100) surface. Therefore, the only non-zero adsorbate reciprocal lattice vectors that lie within the first Brillouin zone of the bare surface are $[ \pm 2 \pi / a, 0]$ and $[0, \pm 2 \pi / a]$, leading to a sum with only four terms. The resulting expression for $R\left(\omega_{0}\right)$ for this system is

$$
R\left(\omega_{0}\right) \approx \frac{m \omega_{0}^{2}}{2 \pi \rho c_{T}^{2} a}\left(2+\frac{1}{1-\left(c_{T} / c_{L}\right)^{2}}\right)=0.0376 .
$$

Using this value, the corrected damping rate is $0.48 \times 10^{12} \mathrm{~s}^{-1}$. Furthermore, the estimated frequency renormalization factor is 0.956 , which, as anticipated, is close to unity. Note that the value of $R\left(\omega_{0}\right)$ was computed using the measured frequency and not the bare frequency. However, since the renormalization is small, this approximation is acceptable. 
In previpus work, we performed an atomistic investigation of this system treating the interactions quantum mechanically. 1 The computed frustrated translational frequency and damping rate were $27 \mathrm{~cm}^{-1}$ and $0.33 \times 10^{12} \mathrm{~s}^{-1}$, respectively. Thus the present elastic continuum theory, the atomistic theory, and the experiments all give a consistent, mutually reinforcing picture of the low-frequency adsorbate vibrational relaxation dynamics; namely, a layer of oscillating adsorbates coupling collectively to substrate phonons at the resonant frequency.

While much research on adsorbed surfaces has focused on ordered overlayer structures, it is also important to consider how the generic case of a disordered or defective overlayer affects the damping rate formula of Eq. (25). For a dense adlayer (as defined above), the relaxation is governed by atomic motions in which all atoms of a given layer oscillate in phase, for both the adlayer and the substrate. Thus the relevant behavior is one-dimensional along the surface-normal direction, and the details of the in-plane structure are not resolved. As a result, the damping rate formula, including its coverage and frequency dependence, is unaffected by disorder in the dense-adlayer limit. In this analysis we have assumed that the adsorbates are uniformly distributed on the surface (i.e., no islands). If islands form, then the local adsorbate density is higher than the average density, and we expect the resonant damping rate to reflect the former value. A more thorough, quantitative treatment of disorder in this problem will be presented in a forthcoming article.

Resonant coupling between an adsorbate overlayer and an elastic substrate provides a damping force for the decay of low-frequency vibrations. We have presented a classical, analytical theory of this mechanism which predicts vibrational damping rates in excellent quantitative agreement with measured values for systems that have been studied experimentally.

Furthermore, we have shown that, even for the smallest measurable coverages, vibrating adsorbates behave collectively when coupling to the substrate. Due to interference effects, a layer of adsorbates oscillating in phase couples only to resonant phonon modes propagating perpendicular to the surface. Only when neighboring adsorbates are separated by distances larger than the wavelength of resonant phonons will new propagation directions be included. The isolated-adsorbate limit, treated by Persson and Ryberg, 2 becomes valid only when many resonant-phonon wavelengths fit between neighboring adsorbate sites. This limit is well below the measurable threshold. Finally, we have also shown that the coupling is strongly coverage dependent. A dense overlayer drives the substrate more efficiently than does a dilute overlayer.

We thank J. P. Toennies for useful discussions during the early stages of this work. Financial support for this project was provided by the National Science Foundation under grants DMR 97-02514 and DMR 93-13047, and by the Petroleum Research Fund of the American Chemical Society under grant 32007-G5.

${ }^{1}$ S. P. Lewis and A. M. Rappe, Physical Review Letters 77, 5241 (1996).

${ }^{2}$ B. N. J. Persson and R. Ryberg, Physical Review B 32, 3586 (1985).

${ }^{3}$ L. D. Landau and E. M. Lifshitz, Theory of Elasticity, 3rd ed. (Pergamon, New York, 1986).

${ }^{4}$ The function $T(\omega)$ in Eq. (14) is the negative of the function $R(\omega)$ from Appendix B of Ref. 2 .

${ }^{5}$ A. A. Maradudin and D. L. Mills, Annals of Physics 100, 262 (1976); and M. G. Cottam and A. A. Maradudin, Surface Excitations, edited by V. M. Agranovich and R. Loudon (Elsevier, New York, 1984).

${ }^{6}$ C. Kittel, Introduction to Solid State Physics, 7th ed. (Wiley, New York, 1996).

7 T. A. Germer, J. C. Stephenson, E. J. Heilweil, and R. R. Cavanagh, Physical Review Letters 71, 3327 (1993); Journal of Chemical Physics 101, 1704 (1994). 